\title{
Teoria Fundamentada Construtivista: características e aspectos operacionais para a pesquisa em enfermagem
}

\author{
Constructivist Grounded Theory: characteristics and operational aspects for nursing research \\ Teoría Fundamentada Constructivista: características y aspectos operacionales para \\ investigación en enfermería
}

Como citar este artigo:

Metelski FK, Santos JLG, Cechine-Peiter C, Fabrizzio GC, Schmitt MD, Heilemann M. Constructivist Grounded Theory: characteristics and operational aspects for nursing research. Rev Esc Enferm USP. 2021;55:e03776. https://doi.org/10.1590/S1980-220X2020051103776

\section{Fernanda Karla Metelski ${ }^{1,2}$ \\ José Luís Guedes dos Santos ${ }^{1}$ \\ Caroline Cechinel-Peiter ${ }^{1}$ \\ Greici Capellari Fabrizzio ${ }^{1}$ \\ Márcia Danieli Schmitt ${ }^{1}$ \\ MarySue Heilemann ${ }^{3}$ \\ ${ }^{1}$ Universidade Federal de Santa Catarina, Programa de Pós-Graduação em Enfermagem, Florianópolis, SC, Brasil. \\ ${ }^{2}$ Universidade Federal de Santa \\ Catarina, Departamento de \\ Enfermagem, Chapecó, SC, Brasil. \\ ${ }^{3}$ University of California, UCLA School of Nursing, Los Angeles, CA, United States.}

Autor correspondente:

José Luís Guedes dos Santos

Campus Reitor João David Ferreira

Lima. Bairro Trindade

CEP 88040-900 - Florianópolis, SC, Brasil

santosjlg29@gmail.com

\begin{abstract}
This is a theoretical analysis of Constructivist Grounded Theory and its application in nursing research. It is organized in three topics: Characteristics of Grounded Theory; Philosophical basis of the constructivist strand; and Constructivist Grounded Theory data analysis particularities. The unique characteristics of Grounded Theory that set it apart from other approaches are simultaneous data collection and analyses, theoretical sampling, constant comparison at each stage of analysis, development of memos for reflexive and comparative analysis, and a coding system with an initial and a focused stage that is flexible and guides the analytical process of building a theory. Before embarking on a Constructivist Grounded Theory study, the literature is reviewed to hone the research question. The philosophical assumptions of symbolic interactionism and social constructivism influence the entire process. Constructivist Grounded Theory emphasizes the construction and interpretation of data recognizing social contexts, interaction, the point of view of participants, and their understandings according to the investigation time, place and situation.
\end{abstract}

\section{DESCRIPTORS}

Grounded Theory; Research; Qualitative Research; Methods; Nursing Methodology Research. 


\section{INTRODUÇÃO}

Como alternativa à pesquisa hipotético-dedutiva, a metodologia da Teoria Fundamentada nos Dados (TFD) surgiu nos Estados Unidos no início dos anos 1960, propondo o desenvolvimento de uma teoria explicativa. A TFD foi criada pelos sociólogos Barney Glaser e Anselm Strauss durante um estudo que examinou a experiência de pacientes terminais. Ao fazer essa investigação, eles questionaram o uso do método científico de verificação e desenvolveram uma maneira inovadora de organizar e analisar dados qualitativos com o objetivo de desenvolver teorias. Como resultado do trabalho de Glaser e Strauss, o método foi descrito com mais detalhes no livro intitulado The Discovery of Grounded Theory: Strategies for Qualitative Research, que lançou as bases da TFD em $1967^{(1-2)}$.

A TFD desafia a visão da metodologia quantitativa como a única forma válida e imparcial de entender a realidade. Glaser e Strauss contestaram a crença de que a pesquisa qualitativa carecia de rigor, criando uma metodologia detalhada para coleta e análise de dados que levou à geração de uma teoria. Apesar do sucesso, os dois autores posteriormente desenvolveram pontos de vista divergentes sobre a aplicação da Teoria Fundamentada e começaram a trabalhar de maneira independente. Cada um seguia uma linha de trabalho específica, dando origem a diferentes vertentes ou perspectivas da $\operatorname{TFD}^{(1-2)}$.

A abordagem de Glaser para a metodologia é conhecida como vertente Glaseriana ${ }^{(3)}$. Glaser estudou na Columbia University, que tinha uma forte tradição positivista; seu trabalho deu origem ao que se denomina TFD objetivista. Nessa perspectiva, os resultados da análise dos dados são considerados verdades que o pesquisador descobre, porém sem levar em consideração os processos de produção desses dados. Ou seja, com a TFD objetivista, o contexto social e a influência do pesquisador não são considerados consequentes, assim como a interação entre o pesquisador e os participantes da pesquisa também não ${ }^{(4)}$. Espera-se que as teorias desenvolvidas com a TFD objetivista prevejam fenômenos futuros e, portanto, surjam como verdades que são descobertas durante a análise de dados brutos ${ }^{(3)}$.

Strauss, em parceria com sua ex-aluna Juliet Corbin, incorporou técnicas de análise de dados, dando origem à vertente straussiana ou relativista da TFD. Strauss estudou na Universidade de Chicago, onde aprendeu sobre o trabalho de campo com os mentores da Chicago School Sociology. Tendo sido influenciado por George Herbert Mead por meio de Herbert Blumer, Strauss baseou-se no pragmatismo e no interacionismo simbólico para refinar sua vertente da TFD. Para Strauss, os pesquisadores fazem trabalho de campo e também se envolvem em reflexões e camadas de análise enquanto se concentram especificamente em ações e processos nos dados ${ }^{(5)}$. Isso possibilita o desenvolvimento de uma teoria ${ }^{(3)}$. Para Strauss, as pessoas são agentes ativos e não vítimas passivas de forças sociais maiores em seu mundo; além disso, o uso da linguagem influencia os significados sociais subjetivos que emergem por meio da ação e, por essas razões, os pesquisadores precisam focar na linguagem e na ação nos dados ${ }^{(4,6)}$.

Na década de 2000, a socióloga Kathleen Charmaz propôs a vertente construtivista da $\mathrm{TFD}^{(3)}$. Ela foi professora de Sociologia na Sonoma State University, na Califórnia, Estados Unidos. No início de sua carreira acadêmica, participou de seminários e workshops ministrados por Barney Glaser e com seu orientador de doutorado em Sociologia, Anselm Strauss, na University of California, em São Francisco, Estados Unidos ${ }^{(5)}$.

Posteriormente, como pesquisadora independente, Charmaz dedicou-se ao desenvolvimento da TFD a partir da perspectiva do construtivismo, às vezes chamada de Teoria Fundamentada em Dados Construtivista (TFDC). Essa vertente da TFD é usada para produzir uma teoria que é considerada um retrato interpretativo da realidade. Nesse sentido, a TFDC propõe que uma teoria seja desenvolvida pelo pesquisador a partir de dados coconstruídos com os participantes, geralmente por meio de entrevistas. Isso significa, segundo Charmaz, que os dados da entrevista são influenciados tanto pelo pesquisador quanto pelo participante, mesmo porque eles são produzidos durante uma entrevista, uma vez que o pesquisador escolhe quais perguntas fazer e interage com o participante à medida que ele responde às perguntas. Posteriormente, a análise dos dados é novamente influenciada pelo pesquisador a partir de sua perspectiva como ser humano que está envolvido com os dados. Com a TFDC, o pesquisador busca compreender os significados atribuídos pelos participantes ao evento ou fenômeno investigado, mas também leva em consideração como a relação entre eles e o participante influencia os próprios dados que coletam. Há ênfase específica no atuar para reduzir os vieses que o pesquisador traz para o processo. O livro Constructing Grounded Theory: A Practical Guide through Qualitative Analysis, de Charmaz, que marca o início da dimensão construtivista, foi publicado em 2006 e teve uma segunda edição em 2014. O livro foi lançado em português em 2009 no Brasil ${ }^{(4-5)}$.

Assim, a perspectiva de TFDC proposta por Charmaz faz parte da chamada $2^{a}$ geração da TFD, que também inclui outras vertentes contemporâneas do método. O principal resultado de um estudo de TFDC é uma teoria desenvolvida a partir da interação do pesquisador com os dados cocriados e a interpretação do pesquisador das experiências e pontos de vista dos participantes do estudo sobre o que eles fazem, sentem, pensam e vivenciam em um determinado contexto. A TFDC possibilita maior flexibilidade na explicação dos processos estudados ${ }^{(1-2)}$. A metodologia da TFD foi ampliada por Charmaz por causa de sua abordagem analítica da TFD, autocrítica na pesquisa, e sua dedicação à mentoria, tanto pessoalmente quanto por meio de seus livros publicados no final dos anos 2000, considerados seu maior legado ${ }^{(7)}$.

$\mathrm{O}$ aspecto aberto da vertente construtivista da TFD tem estimulado seu uso em diversas áreas do desenvolvimento do conhecimento internacional nos últimos anos, incluindo a Ciência da Enfermagem. No Brasil, embora haja um aumento do uso da vertente construtivista, o uso da abordagem straussiana da TFD ainda predomina nas 
pesquisas em enfermagem ${ }^{(8-11)}$. Além disso, estudos nacionais sobre aspectos teóricos e metodológicos da TFD não discutiram especificamente as particularidades da TFDC ${ }^{(8-9,12-13)}$. Portanto, faz-se necessária uma análise teórica das características e aspectos operacionais da vertente construtivista da TFD na pesquisa em enfermagem, principalmente para alunos de pós-graduação e novos pesquisadores interessados na utilização da metodologia.

A presente análise descreve as características e aspectos operacionais da TFDC e sua aplicação na pesquisa em enfermagem.

\section{MÉTODO}

Esta análise crítica é baseada em uma problematização do tema da TFD e inclui uma leitura interpretativa de referências e conceituações relacionadas à TFD. Três tópicos são apresentados: (1) Características da Teoria Fundamentada dos Dados; (2) Base filosófica da Teoria Fundamentada Construtivista; e (3) Particularidades da Teoria Fundamentada Construtivista.

\section{Características da Teoria Fundamentada nos Dados}

Algumas características comuns encontradas em cada uma das principais vertentes da TFD são consideradas centrais para o uso do método: amostragem teórica, coleta e análise simultânea de dados, incluindo a comparação constante de dados, uso de memorandos e desenvolvimento teórico ${ }^{(3-4)}$.

A amostragem teórica é usada quando o pesquisador desenvolveu pelo menos uma categoria analítica provisória. O objetivo da amostragem teórica não é obter capacidade de representar toda uma população ou de generalizar os resultados $^{(4)}$. Em vez disso, o objetivo da amostragem teórica é fornecer ao pesquisador dados adicionais necessários para desenvolver mais plenamente as propriedades de uma determinada categoria. A ausência de detalhes robustos indica a necessidade de coleta de novos dados especificamente para fortalecer e descrever de maneira mais completa as propriedades de uma determinada categoria ${ }^{(4,14-15)}$. Para esclarecer, após o pesquisador criar um roteiro de Entrevistas Semiestruturadas, ele se envolverá na coleta e análise simultânea de dados. Ele fará a codificação linha por linha e a acompanhará com memorandos escritos sobre o que aprendeu acerca do fenômeno até o momento. A codificação da primeira transcrição da entrevista e dos memorandos subsequentes influenciará a maneira como o pesquisador usará seu roteiro de entrevista durante a segunda entrevista. Isso será repetido até que a fase de codificação focalizada comece ${ }^{(4)}$. É durante a análise dos dados que foram agrupados por códigos focalizados que o pesquisador perceberá se a amostragem teórica é necessária.

É importante observar que o conceito de amostragem teórica não difere de acordo com as diferentes perspectivas da TFD. Porém, na vertente construtivista, Charmaz destaca a importância da abstração teórica nesse processo, o que possibilita ao pesquisador identificar fragilidades na descrição das propriedades das categorias em desenvolvimento; pode ser necessária uma análise mais aprofundada para descrever mais completamente as propriedades ${ }^{(16)}$. Por meio da análise e abstração, os dados são elevados de códigos a categorias, e é por meio desse processo que o pesquisador desenvolve a teoria ${ }^{(17)}$.

A coleta de dados é feita simultaneamente com a análise de dados. A comparação constante é uma forma de análise que consiste em comparar dados com dados, códigos com códigos e incidentes com incidentes para construir categorias e articular suas propriedades ${ }^{(4,15,18)}$. Esse trabalho analítico comparativo é feito em todas as etapas da coleta de dados, começando com a primeira transcrição da entrevista. Com essa técnica, o pesquisador obtém uma visão sobre o que está identificando nos dados. Por meio de memorandos analíticos, o pesquisador irá questionar os dados, criticar os códigos e identificar qual categoria teórica os dados representam. Esse processo possibilitará que levem seu trabalho analítico a um nível mais abstrato. Por exemplo, por meio de comparação constante, cada propriedade em desenvolvimento é examinada e rigorosamente analisada ${ }^{(4)}$. Comparações teóricas também podem ser feitas sempre que o pesquisador estiver sobrecarregado de detalhes e precisar distanciar-se para estimular seu pensamento sobre as propriedades e suas dimensões conceituais ${ }^{(6)}$.

Memorandos são registros analíticos informais compostos pelas reflexões, ideias e quaisquer conexões do pesquisador realizadas com base em códigos, inclusive como elas se relacionam umas com as outras. Os pesquisadores que utilizam a TFDC escrevem memorandos antes de começar e enquanto estão coletando dados e durante toda a análise de dados e fases de escrita. O trabalho criativo, analítico e reflexivo realizado por meio de memorandos ajudará a identificar as categorias teóricas e suas propriedades. Em última análise, os memorandos ajudam os pesquisadores a desenvolver a teoria fundamentada. Os memorandos contêm a compreensão do pesquisador sobre sua análise, bem como as descrições mais abstratas e conceituais de cada categoria ${ }^{(4)}$.

Os memorandos também são utilizados para anotações metodológicas para facilitar a compreensão do processo de pesquisa, bem como do fenômeno em estudo ${ }^{(18)}$. Esses registros são tão significativos que auxiliam na condução da pesquisa desde o início até a última fase da TFD, quando as categorias são integradas à formação da teoria ${ }^{(4)}$. A construção dos memorandos pode ser manuscrita, digitada ou inserida em softwares de gerenciamento de dados (aqueles que organizam os dados), dependendo da preferência e organização do pesquisador.

As teorias podem ser substantivas ou formais, dependendo da extensão e do alcance do estudo. Em um estudo de situação específica, é desenvolvida uma teoria substantiva que pode ser aplicada a um determinado campo e, para isso, a teoria tem um alcance localizado. No entanto, quando os achados são mais robustos em uma pesquisa mais extensa com um escopo mais amplo, uma teoria formal pode ser desenvolvida ${ }^{(4,14-15)}$. Teorias substantivas formam a base para o desenvolvimento de uma teoria formal; entretanto, estudos futuros adicionais são necessários para desenvolvê-las ainda melhor ${ }^{(4,14)}$. 


\section{BASE FILOSÓFICA DA TFD CONSTRUTIVISTA}

Como já foi mencionado, a TFDC supõe que os dados são coconstruídos por meio da interação pesquisador-participante. Além disso, as análises e os produtos das análises (categorias, suas propriedades e as relações entre as categorias) são desenvolvidos por meio da interação do pesquisador com os dados. A TFDC parte do pressuposto de que o pesquisador não é neutro, pois é um ser humano com sua maneira própria de usar a linguagem, suas próprias opiniões e valores. No entanto, o pesquisador deve fazer um trabalho reflexivo para reduzir a influência de seus vieses no participante e na coconstrução dos dados, bem como na sua análise dos dados. O construcionismo social é uma postura filosófica que supõe que todas as pessoas assumem a realidade como a percebem; portanto, é uma construção social, enquanto os rigores da metodologia da TFD, incluindo a TFDC, destinam-se a orientar o pesquisador a fazer o trabalho reflexivo e analítico necessário para priorizar ao máximo a perspectiva dos participantes ${ }^{(3-4,15,19)}$. A TFDC enfatiza especificamente que qualquer pesquisa realizada por seres humanos inevitavelmente apresentará um retrato interpretativo do mundo. Assim, a vertente construtivista difere da versão original da TFD de Glaser e Strauss, que afirmam que a teoria é descoberta nos dados e, quando localizada, não é influenciada pelo envolvimento do pesquisador ${ }^{(4,20)}$. Por essas razões, a TFDC pode ser considerada uma inovação da TFD.

Os fundamentos filosóficos da TFD vêm do Interacionismo Simbólico (IS) e do construtivismo social. George Herbert Mead desenvolveu o IS, uma teoria abstrata sobre interação que foi aperfeiçoada por Herbert Blumer na Universidade de Chicago. O IS pressupõe que a interação é inerentemente dinâmica e que todos os seres humanos se envolvem em um processo interpretativo na vida diária. Portanto, o IS aborda como as pessoas criam, interpretam, endossam ou alteram significados com base em ações e interações vivenciadas em suas vidas diárias ${ }^{(21)}$. No IS, todas as pessoas são consideradas participantes ativos engajados no mundo em que vivem; é por meio da interação com outras pessoas e consigo mesmas (chamada ação mental) que as pessoas dão sentido ao mundo ${ }^{(22)}$.

A TFDC está situada na tradição interpretativa em que os pesquisadores pressupõem que dados e análises são construções sociais e que participantes e pesquisadores igualmente constroem significados. Portanto, pressupõe-se que qualquer teoria desenvolvida por meio de pesquisa é uma interpretação que reflete não apenas as experiências dos participantes, mas também a percepção do pesquisador sobre o fenômeno. Um estudo de TFDC é influenciado por como, quando e em que medida a experiência descrita em uma teoria integra posturas, situações e relacionamentos mais $\operatorname{amplos}^{(4)}$. A metodologia possibilita conceituar as ações dos participantes e os processos de suas experiências vividas ${ }^{(5)}$.

Os conceitos do IS são compatíveis com o construtivismo e são úteis para ajudar o pesquisador a refletir sobre suas percepções do mundo. O IS pressupõe que indivíduos e grupos interagem e, por meio dessas interações, surge o significado. Para o pesquisador, os conceitos abstratos do
IS frequentemente permanecem em segundo plano em sua consciência até que se tornem relevantes durante a codificação ou análise de dados. Os conceitos do IS são ferramentas abstratas que se tornam úteis para analisar processos sociais em dados pois enfatizam a interação como uma forma crucial de ação social ${ }^{(23)}$.

Nesse sentido, o IS afirma que as pessoas são seres sociais que estão envolvidos na interação com os outros, com eles próprios e com seu ambiente. Por meio dessas interações, incluindo a comunicação com os outros e a reflexão constante sobre as próprias ações, o significado emerge e contribui para a forma como a pessoa percebe a realidade ${ }^{(4,24)}$. O IS tem três princípios-chave: o primeiro é que as pessoas agem de acordo com o significado que uma determinada situação tem para elas; o segundo princípio indica que o significado não é inerente ao fato, mas é atribuído por meio da interação social contínua ${ }^{(25)}$; e, finalmente, esses significados atribuídos não são estanques, mas podem ser redefinidos por meio de processos de reflexão e interpretativos ${ }^{(14,24)}$. A experiência e o comportamento humanos são conceitos complexos e instáveis, guiados por símbolos e significados baseados nas interações com o meio ambiente e consigo mesmo ${ }^{(24)}$.

Os fundamentos do construtivismo e do IS, como os fundamentos filosóficos da TFD, influenciam a maneira como os pesquisadores são orientados para a noção de realidade, incluindo o que e como ela pode ser conhecida. No entanto, é crucial esclarecer que o IS não é um referencial teórico inerente a uma teoria fundamentada que um pesquisador desenvolverá; em vez disso, informa o pesquisador e aumenta sua capacidade de usar o método ${ }^{(26)}$.

Muitos estudos da TFDC não utilizam uma estrutura filosófica/teórica, mas quando o fazem, o IS prevalece, embora não seja a única que pode influenciar um pesquisador que usa a TFDC ${ }^{(18,27)}$. No Brasil, outra referência comumente utilizada é o Paradigma da Complexidade, cujo principal proponente é Edgar Morin. Essa estrutura é útil para estudos de processos, porque o conhecimento e as relações humanas não são simplesmente lineares. Em vez disso, são complexos, multifacetados e estão constantemente em construção ${ }^{(11)}$.

\section{PARTICULARIDADES DA TEORIA FUNDAMEN- TADA CONSTRUTIVISTA}

\section{USO DA LITERATURA: EM TODAS AS ETAPAS E COMPILADO AO FINAL}

É importante rever a literatura antes de realizar uma TFDC. Conhecer o estado da arte em relação a um fenômeno possibilita situar a questão de pesquisa específica para o estudo em questão. A literatura pode servir para defender a posição do pesquisador. Possibilita ao leitor identificar as razões pelas quais o estudo está sendo feito. Uma revisão da literatura antes de um estudo de TFDC envolve pesquisas publicadas e outros referenciais teóricos ${ }^{(4,27)}$.

Após a realização dessa compilação, tipicamente a literatura não é revisada novamente até a análise dos dados, para não interferir na criatividade do pesquisador ${ }^{(3)}$. Depois da análise dos dados, uma crítica ao trabalho publicado ajudará 
a posicionar os achados do estudo de TFDC em relação ao conhecimento existente. Isso possibilita a comparação dos resultados de outros estudos e outras teorias, podendo ajudar a esclarecer o conteúdo das categorias teóricas desenvolvidas e demonstrar como a teoria acrescenta ou vai além do conhecimento existente ${ }^{(4,20)}$.

$\mathrm{Na}$ abordagem clássica da TFD, a revisão bibliográfica é adiada para evitar que ideias pré-concebidas influenciem o pensamento dos pesquisadores ou previnam a tendência de ajustar conceitos já existentes às narrativas dos participantes. $\mathrm{Na}$ TFDC, essa postura é considerada um excesso, uma vez que todos possuem conhecimentos prévios que inegavelmente influenciam na interpretação dos dados. Além disso, as teorias existentes sensibilizam os pesquisadores, ou fornecem sensibilidade teórica, que se refere à capacidade que o pesquisador tem de reconhecer variações nos dados, de ter insights e interpretá-los, identificando dados relevantes para o estudo e equilibrando ciência e criatividade ${ }^{(4,18)}$.

Com a TFDC, o uso da literatura publicada contribui para a argumentação e credibilidade do trabalho. Embora uma revisão inicial da literatura possa ter preparado o pesquisador e ajudado a definir o foco de seu trabalho por meio de estudos-chave e do desenvolvimento do argumento que levou à questão da pesquisa, somente muito tarde no processo ou após a análise dos dados ser concluída é que se percebe a diferença entre essa TFD e as teorias anteriores ${ }^{(4)}$.

\section{Sistema de CODIFICAÇÃO: ABERTO PARA CONSTRUÇÃO DE TEORIA}

A codificação é um primeiro passo importante na análise de dados, que ajuda o pesquisador a se concentrar na ação e nos processos dos dados e nos significados que cada participante atribui aos fatos e às experiências vivenciadas. Por meio da codificação, o pesquisador busca uma compreensão mais abstrata dos dados para articular o que está acontecendo com o tempo, lugar e situação do participante ${ }^{(4)}$. Os segmentos de dados são rotulados e, posteriormente, classificados em grupos para que possam ser categorizados para produzir uma interpretação explicativa do fenômeno; Charmaz afirmou que "é o elo fundamental entre a coleta de dados e o desenvolvimento de uma teoria emergente para explicar esses dados"(4). Os códigos criados por pesquisadores fornecem descrições gerenciáveis de experiências vividas expressas em uma frase curta de palavras (usando a forma de gerúndio dos verbos). A partir disso, os pesquisadores podem desenvolver e, então, tecer afirmações teóricas que respeitem o contexto de cada participante à medida que as análises prosseguem ${ }^{(4)}$.

O sistema de codificação da TFDC envolve pelo menos duas etapas hierárquicas: 1) codificação inicial e 2) codificação focalizada ${ }^{(21)}$. A codificação inicial possibilita ao pesquisador familiarizar-se mais com os dados em um nível granular. Nessa etapa, a codificação envolve cada palavra, linha ou segmento de dados; os pesquisadores focam nos dados e na realidade vivenciada pelo participante da pesquisa $^{(4,15)}$. Portanto, sugere-se que sejam traçados códigos, permanecendo-se próximo aos dados e com foco no que está acontecendo para o participante em cada segmento. A codificação in vivo(4) é um tipo particular de codificação inicial que mantém as próprias palavras dos participantes, geralmente porque a frase exata declarada por um participante expressa sucintamente uma grande quantidade de significado. Esses códigos identificam as declarações explícitas feitas por um participante e chamam a atenção para preocupações implícitas. Ambos podem ser explorados mais profundamente em memorandos. O pesquisador deve estar aberto a todas as orientações teóricas sugeridas pela leitura dos dados. Assim, os códigos são considerados provisórios. Eles podem ser reformulados para uma compreensão mais completa ou para condensar os significados e as ações dos participantes em uma linguagem mais concisa ${ }^{(4)}$.

A codificação focalizada possibilita identificar categorias provisórias, resumir e explicar segmentos maiores de dados. A codificação focalizada é baseada em dados, mas envolve o agrupamento de códigos e ideias iniciais. $\mathrm{Na}$ etapa de codificação focalizada, os códigos iniciais mais significativos ou frequentes são identificados e então usados para agrupar os códigos iniciais em grupos mais focados. Para fazer isso, os códigos iniciais são comparados para possibilitar uma maior compreensão analítica de como os dados podem ser agrupados; isso levará à capacidade de explicar segmentos de dados maiores ${ }^{(4,15)}$.

Os códigos iniciais que o pesquisador identifica como os que aparecem com mais frequência na codificação de dados e aqueles que se destacam e são selecionados pelo pesquisador como códigos focalizados. Em seguida, esses códigos são usados para marcar todo o conjunto de dados e os dados codificados são classificados em grupos com base em um determinado código focalizado. Neste momento, a comparação de códigos com códigos e dados com dados, dentro de cada grupo de código focalizado, é mais uma vez crucial na análise. Várias técnicas analíticas são usadas para investigar cada grupo de código focalizado para identificar características que se destacam e têm um significado maior. A redação de memorandos continua sendo especialmente importante durante o estágio de codificação focalizada. A análise dentro de cada grupo de código focalizado provavelmente inspirará vários memorandos que documentam e rastreiam o trabalho analítico. $\mathrm{O}$ pesquisador pode usar memorandos para documentar a maneira como seguiram pistas para entender, por exemplo, uma sequência que se tornou evidente nos dados, ou para identificar padrões nos dados, ou para explorar palpites sobre a resposta à pergunta “o que está acontecendo aqui?” examinando dados dentro de um grupo de código focalizado de vários participantes ${ }^{(4,21)}$.

A codificação teórica ocorre mais tarde no estágio de codificação focalizada, depois que as categorias provisórias e suas propriedades foram desenvolvidas. A codificação teórica pode trazer clareza aos códigos focalizados e pode ajudar o pesquisador a contar uma história analítica mais coerente por meio de seus resultados ${ }^{(4)}$. No entanto, codificação teórica não é o mesmo que amostragem teórica (ou seja, coletar novos dados para trazer os detalhes necessários para que uma propriedade de uma categoria atinja a saturação). A codificação teórica envolve a aplicação de vários esquemas analíticos para elevar o nível de abstração e aproximar os produtos analíticos da formação de uma teoria. Isso trará 
coerência, pois ajuda o pesquisador a identificar as relações entre categorias, ou entre conceitos completamente desenvolvidos, em uma teoria fundamentada $a^{(4)}$.

A codificação na TFDC é um sistema aberto, ou seja, os códigos são criados durante a análise dos dados. Ambos são produto da análise e também constituem o processo de análise. A interação ativa do pesquisador com os dados resulta no desenvolvimento de códigos que são analisados por comparação. Assim, na TFDC, os códigos pré-concebidos não são utilizados ${ }^{(3-4)}$. Embora os códigos sejam informados pela realidade empírica, é o pesquisador quem escolhe as palavras que os compõem. Enquanto o pesquisador de TFDC busca compreender as experiências e percepções dos participantes por meio da codificação, nunca se deve esquecer que os códigos são criados pelo pesquisador e são influenciados por sua perspectiva ${ }^{(4)}$.

$\mathrm{O}$ processo de codificação é o nome dado à codificação feita usando a forma de gerúndio dos verbos (forma nominal do verbo associado ao sufixo -ndo). $\mathrm{O}$ processo de codificação é especialmente importante na TFDC porque o pressuposto do IS é que todo participante seja um ator, não uma vítima, de sua vida cotidiana ${ }^{(25)}$. Códigos são palavras que descrevem as ações de uma pessoa, por exemplo: buscando, realizando, sentindo, culpando, esperando obtendo, negando, lutando, etc. O objetivo da codificação do processo é reduzir a tendência de projetar uma interpretação muito cedo no processo de análise. Além disso, é muito fácil para os pesquisadores, como seres humanos, projetar significado "sobre" um participante por meio de um código aplicado a dados sem atenção cuidadosa ao participante, um ator, que se envolve com o mundo no contexto de sua própria experiência vivida. Com a codificação do processo, o pesquisador ganha uma ferramenta que auxilia no desenvolvimento da sensibilidade teórica, pois o foco está no que está acontecendo no mundo do participante, para ele, com ele e sobre ele em toda e qualquer linha de $\operatorname{dados}^{(4)}$. O Quadro 1 resume as etapas de codificação.

Quadro 1 - Resumo das etapas de codificação da TFD construtivista.

\begin{tabular}{|c|c|c|}
\hline Tipo & Foco & Etapas \\
\hline Codificação Inicial & $\begin{array}{c}\text { Identificar a ação nas } \\
\text { palavras dos participantes, } \\
\text { incluindo intenções e } \\
\text { experiências. }\end{array}$ & $\begin{array}{l}\text { - Analisar os dados linha por linha. } \\
\text { - Criar códigos de processo usando verbos que respondem "o que está acontecendo aqui?". } \\
\text { - Desenvolver códigos in vivo usando as próprias palavras dos participantes, conforme } \\
\text { declarado. }\end{array}$ \\
\hline $\begin{array}{l}\text { Codificação } \\
\text { focalizada }\end{array}$ & $\begin{array}{l}\text { Identificar os códigos iniciais } \\
\text { mais frequentes e mais } \\
\text { importantes e usá-los para } \\
\text { agrupar dados para uma } \\
\text { análise mais profunda. }\end{array}$ & $\begin{array}{l}\text { - Criar códigos focalizados com base nos códigos mais significativos ou frequentes. } \\
\text { - Marcar todo o conjunto de dados com códigos focalizados e, em seguida, usá-los para } \\
\text { agrupar os dados de acordo. } \\
\text { - Analisar os dados em cada grupo de código focalizado usando várias técnicas, incluindo } \\
\text { codificação teórica. } \\
\text { - Refinar os códigos focalizados em categorias provisórias. } \\
\text { - Desenvolver as propriedades de cada categoria, até que a saturação seja atingida (ou seja, } \\
\text { até que cada propriedade de cada categoria seja totalmente descrita; pode ser necessária } \\
\text { uma amostragem teórica para isso) }{ }^{(4)} \text {. }\end{array}$ \\
\hline
\end{tabular}

O objetivo da TFD é desenvolver conceitos que se organizem em uma teoria para descrever e explicar um fenômeno até então pouco compreendido no mundo empírico. $\mathrm{O}$ trabalho analítico da TFDC orienta os pesquisadores a identificar relações entre conceitos desenvolvidos a partir de dados codificados após análise cuidadosa, em etapas e rigorosa ${ }^{(21,28)}$. A saturação das categorias é obtida por meio de análises de dados. A amostragem teórica é feita para obter dados adicionais para mais análises, de forma que as propriedades de cada categoria sejam totalmente descritas; isso é feito quando necessário para saturar as propriedades. Se os dados e as análises forem robustos, uma categoria pode ser elevada ao nível de um conceito. As ferramentas analíticas da codificação teórica são utilizadas na fase final para identificar as relações entre os conceitos para formar uma teoria.

Este processo envolve não apenas a indução, mas também um processo denominado abdução. Com a abdução, o pesquisador vai e volta entre os dados e as possíveis interpretações dos dados, buscando chegar à explicação teórica mais plausível. Por meio desse processo, o pesquisador testa as hipóteses, confirmando ou não as ideias ${ }^{(4,15)}$. A Figura 2 ilustra o desenvolvimento de uma teoria a partir de dados codificados ao se utilizar TFDC. É uma versão modificada do diagrama originalmente desenhado por Saldaña ${ }^{(28)}$. Ele descreve novamente como a TFDC é conduzida, começando com dados que são subsequentemente codificados e analisados para formar categorias com propriedades.

Depois que uma teoria é desenvolvida e refinada, ela pode ser divulgada. É nesse momento que os leitores avaliarão a utilidade da teoria e, consequentemente, determinarão o valor da $\mathrm{TFD}^{(4)}$. Vários critérios de avaliação têm sido utilizados para criticar a TFD, mas na vertente construtivista destacam-se a credibilidade, originalidade, ressonância e utilidade $^{(4,15)}$. Credibilidade refere-se a se as conexões lógicas entre os dados e as interpretações foram feitas ou não, cobrindo uma ampla variedade de observações empíricas. A originalidade sinaliza que a pesquisa produziu novos conhecimentos sobre a realidade interpretada dos participantes 
e depende do valor de sua contribuição teórica e social. A ressonância exige que a TFD tenha um nível de completude da experiência estudada que revele significados pressupostos que façam sentido para os participantes ou para as pessoas que compartilham as mesmas experiências. Por fim, a utilidade avalia se a pesquisa tem impacto, melhora o dia a dia das pessoas, contribui para um mundo melhor e incentiva novas investigações ${ }^{(4)}$.

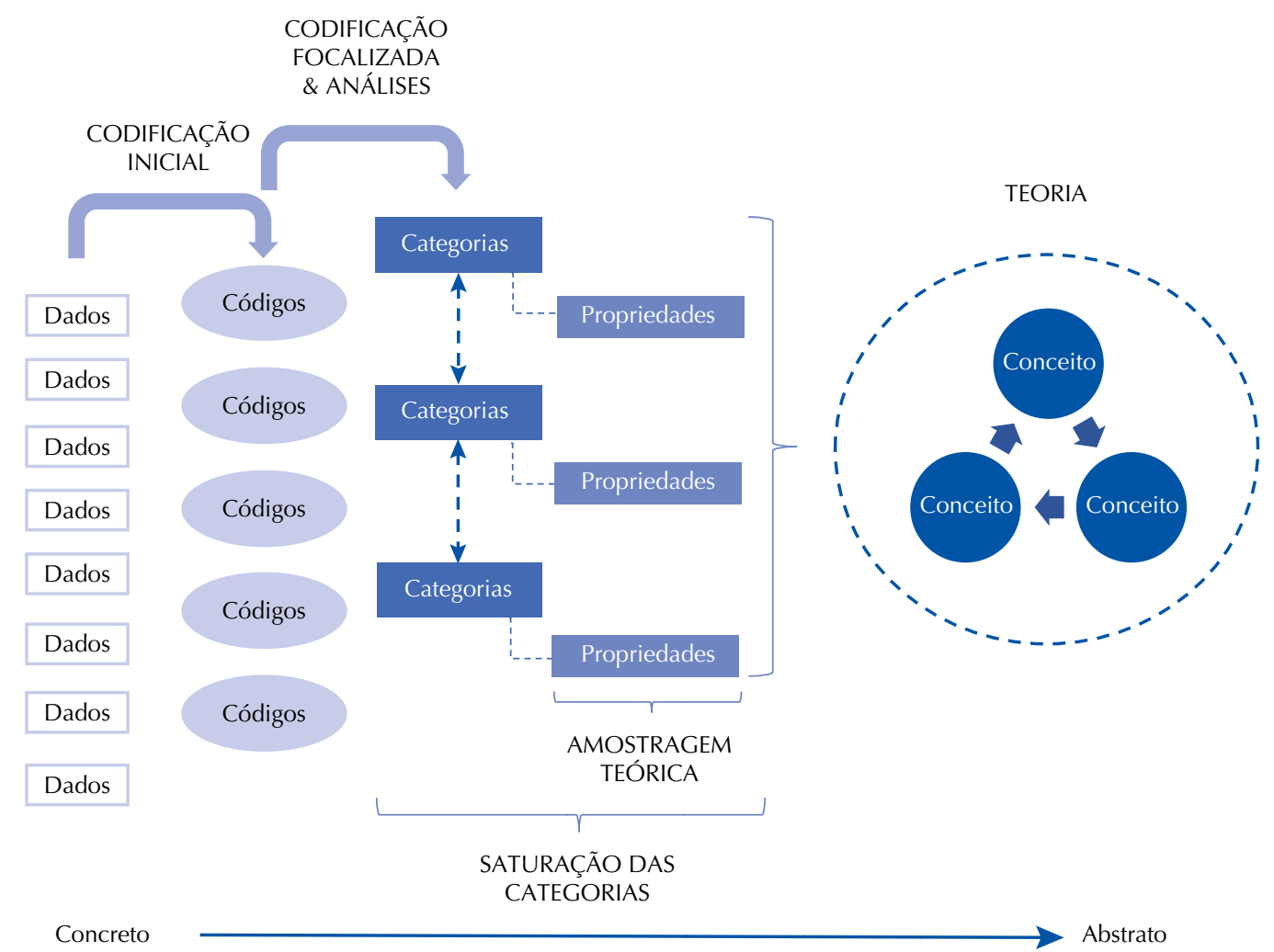

Adaptado de: Saldaña, 2015(28).

Figura 1 - Processo de TFDC para desenvolver teoria a partir de dados codificados.

\section{CONSIIDERAÇÕES FINAIS}

A vertente Construtivista da TFD foi desenvolvida por Kathy Charmaz como uma nova interpretação desta metodologia. Evoluiu das vertentes Clássica e Straussiana da TFD e, por esta razão, a TFDC tem características em comum com estas vertentes: amostragem teórica, coleta e análise simultâneas de dados incluindo a comparação constante de dados, a utilização de memorandos e o desenvolvimento de teorias. Nesse sentido, cabe destacar que, independentemente da abordagem específica, a utilização da TFD exige que os pesquisadores atendam aos preceitos que conferem qualidade e rigor metodológico aos seus estudos e que diferenciem a TFD de outras metodologias de pesquisa qualitativa.

Dentre as características que diferenciam os aspectos da TFD estão os fundamentos filosóficos, o uso da literatura e o sistema de codificação da análise de dados. Os fundamentos da TFD mais frequentemente invocados advêm do IS, que afirma que o significado emerge das ações e interações entre os indivíduos e contribui para a maneira como eles percebem a realidade.

$\mathrm{Na}$ TFDC, o uso da literatura publicada é recomendado antes da implementação do estudo e após o desenvolvimento da teoria fundamentada. Ao revisar pesquisas publicadas e outros referenciais teóricos, o pesquisador desenvolve a questão de pesquisa. Posteriormente, os achados de outros estudos que corroboram, são suporte ou se opõem à interpretação dos dados são valiosos para situar a teoria fundamentada no contexto do estado da ciência.

A codificação, uma parte crucial da análise de dados em TFDC, compreende uma fase inicial e uma fase focalizada. O sistema de codificação é aberto e não pré-determinado. Assim, o pesquisador desenvolve códigos a partir de dados e usa memorandos para explorar palpites e, por isso, é necessário que o pesquisador esteja aberto para explorar todos os possíveis rumos teóricos. A escolha das palavras que compõem os códigos reflete a perspectiva do pesquisador sobre as experiências ou percepções dos participantes e suas perspectivas. Em última análise, teorias baseadas em dados de um estudo de TFD são construídas por meio da interação entre pesquisadores e participantes, trazendo um retrato interpretativo da realidade.

Esta análise tem o potencial de aprofundar a compreensão da TFD como metodologia. Assim, pode contribuir para a utilização desta metodologia em futuras pesquisas em Enfermagem no Brasil. 
RESUMO

Análise teórica da Teoria Fundamentada Construtivista e sua aplicação na pesquisa em enfermagem. Está organizada em três tópicos: características da Teoria Fundamentada nos Dados; base filosófica da vertente construtivista; e particularidades da análise de dados da Teoria Fundamentada Construtivista. As características exclusivas da Teoria Fundamentada que a diferenciam de outras abordagens são coleta e análise simultâneas de dados, amostragem teórica, comparação constante em cada estágio de análise, desenvolvimento de memorandos para análise reflexiva e comparativa e um sistema de codificação com uma etapa inicial e uma focalizada que é flexível e orienta o processo analítico de construção de uma teoria. Antes de embarcar em um estudo da Teoria Fundamentada Construtivista, a literatura é revisada para aprimorar a questão de pesquisa. Os pressupostos filosóficos do interacionismo simbólico e do construtivismo social influenciam todo o processo. A Teoria Fundamentada Construtivista enfatiza a construção e interpretação de dados reconhecendo contextos sociais, interação, o ponto de vista dos participantes e seus entendimentos de acordo com o tempo, lugar e situação da investigação.

\section{DESCRITORES}

Teoria Fundamentada; Pesquisa; Pesquisa Qualitativa; Métodos; Pesquisa Metodológica em Enfermagem.

\section{RESUMEN}

Análisis teórico de la Teoría Fundamentada Constructivista y su aplicación en investigación en enfermería. Se organiza en tres temas: Características de la Teoría Fundamentada; Base Filosófica de Vertiente constructivista; y particularidades del análisis de datos de la teoría fundamentada constructivista. Las características exclusivas de la Teoría Fundamentada que la difieren de otros abordajes son colecta y análisis simultáneos de datos, muestra teórica, comparación constante en cada etapa de análisis, desarrollo de memorandos para análisis reflexivos y comparativos y un sistema de codificación con una etapa inicial, y una enfocada, que es flexible y que orienta el proceso analítico de construcción de una teoría. Antes de empezar en un estudio de la Teoría Fundamentada Constructivista, se revisa la literatura para perfeccionar la cuestión de investigación. Los presupuestos filosóficos del interaccionismo simbólico y del constructivismo social influyen en todo el proceso. La Teoría Fundamentada Constructivista tiene énfasis en la construcción e interpretación de datos que reconocen contextos sociales, interacción, el punto de vista de los participantes y su comprensión de acuerdo con el tiempo, lugar y situación de investigación.

\section{DESCRIPTORES}

Teoría Fundamentada; Investigación; Investigación Cualitativa; Métodos; Investigación Metodológica en Enfermería.

\section{REFERÊNCIAS}

1. Apramian T, Cristancho S, Watling C, Lingard L. (Re)Grounding grounded theory: a close reading of theory in four schools. Qual Res. 2017;17(4):359-76. https://doi.org/10.1177/1468794116672914

2. Tie YC, Birks M, Francis K. Grounded theory research: a design framework for novice researchers. SAGE Open Med. 2019;7(2):1-8. https:// doi.org/10.1177/2050312118822927

3. Santos JL, Cunha KS, Adamy EK, Backes MT, Leite JL, Sousa FG. Data analysis: comparison between the different methodological perspectives of the Grounded Theory. Rev Esc Enferm USP. 2018;52:e03303. https://doi.org/10.1590/s1980-220x2017021803303

4. Charmaz K. A construção da teoria fundamentada: guia prático para análise qualitativa. Porto Alegre: Artmed; 2009.

5. Charmaz K, Keller R. A personal journey with grounded theory methodology. Forum Qual Soc Res. 2016;17(1): https://doi.org/10.17169/ fqs-17.1.2541

6. Corbin J, Strauss A. Basics of qualitative research: techniques and procedures for developing Grounded Theory. Thousand Oaks: Sage; 2015.

7. Morse J. A moment of silence. Qual Health Res. 2020 Nov;30(13):1985-8. https://doi.org/10.1177/1049732320958713

8. Silva GW, Enders BC, Sousa FG, Sena JF, Santos RC, Silva AB. Grounded theory in theses and dissertations of Brazilian nursing. Texto Contexto Enferm. 2018;27(4):e3870017. https://doi.org/10.1590/0104-07072018003870017

9. Peiter CC, Santos JL, Kahl C, Cunha KS, Lacerda MR. Grounded theory: use in scientific articles published in brazilian nursing journals with qualis A classification. Texto Contexto Enferm. 2020;29:e20180177. https://doi.org/10.1590/1980-265x-tce-2018-0177

10. Koerich C, Erdmann AL, Lanzoni GM. Professional interaction in management of the triad: Permanent Education in Health, patient safety and quality. Rev Latino-Am Enferm 2020;28:e3379. https://doi.org/10.1590/1518-8345.4154.3379

11. Girardon-Perlini NM, Simon BS, Lacerda MR. Grounded Theory methodological aspects in Brazilian nursing thesis. Rev Bras Enferm. 2020;73(6):e20190274. https://doi.org/10.1590/0034-7167-2019-0274

12. Dantas CC, Leite JL, Lima SB, Stipp MA. Grounded theory: conceptual and operational aspects: a method possible to be applied in nursing research. Rev Latino-Am Enferm 2009;17(4):573-579. https://doi.org/10.1590/S0104-11692009000400021

13. Koerich C, Copelli FH, Lanzoni GM, Magalhães AL, Erdmann AL. Grounded theory: evidencing divergences and ontributions for nursing research. REME. 2018;22:e-1084. http://www.dx.doi.org/10.5935/1415-2762.20180014

14. Kenny M, Fourie R. Contrasting classic, straussian, and constructivist grounded theory: methodological and philosophical conflicts. Qual Rep. 2015;20(8):1270-89. https://doi.org/10.46743/2160-3715/2015.2251

15. Rieger KL. Discriminating among grounded theory approaches. Nurs Inq. 2019 Jan;26(1):e12261. https://doi.org/10.1111/nin.12261

16. Davoudi N, Nayeri ND, Raiesifar A, Poortaghi S, Ahmadian S. Issues of theoretical sampling: a narrative review. Nurs Midwifery Stud. 2016;6(2):e38284. https://doi.org/10.5812/nmsjournal.38284

17. Hoare K, Ward K, Walker R. English children's respectful reflections of the rights and lives of their kenyan peers: a grounded theory study. Child Soc. 2018;32(2):145-55. https://doi.org/10.1111/chso.12244

18. Barreto MS, Vivar CG, Marcon SS. Methodological quality of Grounded Theory research with families living with chronic illness. Int J Afr Nurs Sci. 2018;8:14-22. https://doi.org/10.1016/j.ijans.2018.01.001 
19. Zocche DA, Adamy EK, Lima CF. Perspectiva construtivista da teoria Fundamentada nos dados. In: Lacerda MR, Santos JL, editors. Teoria fundamentada nos dados: bases teóricas e metodológicas. Porto Alegre: Moriá; 2019. p. 105-127.

20. Goudind C. Navigating the Complexities of Grounded Theory Research in Advertising. J Advert. 2017;46(1):61-70. https://doi.org/10.10 80/00913367.2017.1281775

21. Charmaz K. "With constructivist grounded theory you can't hide": social justice research and critical inquiry in the public sphere. Qual Inq. 2020;26(2):165-76. https://doi.org/10.1177/1077800419879081

22. MacDonald M, Schreiber RS. Constructing and deconstructing: grounded theory in a postmodern world. In: Schreiber RS, Stern PN, editors. Using grounded theory in nursing. New York: Springer; 2001. p. 35-54.

23. Bakker JI. Grounded theory methodology and grounded theory method: introduction to the special issue. Sociol Focus. 2019;52(2):91-106. https://doi.org/10.1080/00380237.2019.1550592

24. Lee Y. Patients' perception and adherence to vaginal dilator therapy: a systematic review and synthesis employing symbolic interactionism. Patient Prefer Adherence. 2018;12:551-60. https://doi.org/10.2147/PPA.S163273

25. Blumer H. Symbolic interactionism: perspective and method. Englewood Cliffs: Prentice Hall; 1969.

26. Aldiabat KM, Le Navenec C. Data saturation: the mysterious step in grounded theory methodology. Qual Rep. 2018;23(1):245-61. https:// doi.org/10.46743/2160-3715/2018.2994

27. Gomes IM, Hermann AP, Wolff LDG, Peres AM, Lacerda MR. Grounded theory in nursing: an integrative review. Rev Enferm UFPE On Line. 2015;9(1):466-474. https://doi.org/10.5205/reuol.5221-43270-1-RV.0901 supl201527

28. Saldaña J. The coding manual for qualitative researchers. London: Sage; 2015.

\section{Apoio financeiro}

Coordenação de Aperfeiçoamento de Pessoal de Nível Superior (Capes). Código 001. Conselho Nacional de Desenvolvimento Científico e Tecnológico (CNPq). 